\title{
The Analysis on the Relationship Between Daily Stress and Troubled Sleep
}

\author{
Jiahan Wang ${ }^{1, *}$ \\ ${ }^{1}$ Qingdao No.2 Middle School of Shandong Province, Qingdao, Shandong, China \\ *Corresponding author's e-mail:2205345275@qq.com
}

\begin{abstract}
Troubled sleep is increasingly prevalent among people in different age groups. Thus, understanding the causes and coping methods of troubled sleep is very important. This study only focused on the effects of stress on troubled sleep and used PMData to analyze the relationship between stress and troubled sleep. By applying Pearson product-moment correlation coefficients and simple linear regression model, this paper found the stress can lead to troubled sleep (poor sleep and shortened sleep). The stress can lead to physical effects such as raised blood pressure as well as unpleasant mental effects. These effects can make people hard to stop thinking about stressful moment and fall asleep. Lack of sleep leads to increased levels of cortisol and other stress hormones that regulate brain function, making sleep worse and stress greater, which is like a vicious circle. It is hoped that the relationship found between stress and troubled sleep can be useful to improve the effectiveness of the coping methods to troubled sleep.
\end{abstract}

Keywords: Daily tress, sleep quality, sleep duration, troubled sleep.

\section{INTRODUCTION}

People spend at least one third of their lives sleeping, which reveals the importance of sleep to persons in all age groups. However, millions of American across all demographic groups are experiencing sleep disorders. According to various surveys and studies, $70 \%$ of adults live with insufficient sleep or rest at least once each month [1], and chronic insomnia affects 10\%-30\% of adults [2]. Inadequate sleep is associated with increasing disease risks. A weakened immune system and disability to resist disease as well as recover are closely related to poor sleep. People with sleep deficiency are more likely to have underlying illnesses, such as cardiovascular, cerebrovascular, hypertension and other diseases [3]. Along with the prevalence of sleep deficiency among different sleeper groups and the importance of highquality sleep to people, there has been an upper trend in the interest in exploring the causes of poor sleep. Prior research has suggested that chronic medical conditions, frequent use of medications, social distance, unhealthy living habits as well as various stress can lead to sleep deficiency. This paper aims at analyzing the relationship between daily stress and troubled sleep. It is hoped that this research can further raise public awareness towards the current pervasive problems in sleep and help to improve the therapeutic strategies to cope with this type of sleep deficiency in order to enhance public health and well-being.

\section{LITERATURE REVIEW}

Because of the importance of sleep and the prevalence of sleep deficiency, there are several studies of the association between sleep and stress with different concerns. Prior research indicates that the stress from work is associated with sleep. By using logistic regression analysis, some studies have found that people's work stress caused by frequent interference or worry at work can lead to troubled sleep [4]. High work demands contribute to higher risks of poor sleep [5]. Having greater work-related stress, people are more likely not to stop thinking about work during free time, even during the time before sleep. Therefore, people experience more time awaking and feel hard to fall asleep. Like the relationship between work-related stress and sleep, stress from social relationship leads to troubled sleep as well. Studies of family relationships and troubled sleep have found that compared with family relationships which family members provide support with each other, having strained family relationship is riskier to poor sleep, even with more frequent contacts [6]. There are some studies which suggest that the control over stressful events also plays an effective mediating role in the association 
between sleep and stress. Effective coping skills can reduce the stressful feelings, which further improve the sleep quality [7]. In a nutshell, the sources of stress are various which can be from work, family relationship, etc. Stress does lead to poor sleep. The more people feel stressful, the greater risk of troubled sleep people experience.

\section{WHAT IS DAILY STRESS}

Daily stress is mundane annoyances caused by routine daily activities which can be divided into two categories. The classification of daily stress is determined by predictability, so there are anticipated daily stressors like working long hours as well as preparing for the exams and unanticipated daily stressors such as having arguments with families. Although daily stress is common throughout our lives and is relatively minor, it may still potentially affect our normal everyday lives.

\section{HOW STRESS AFFECTS SLEEP}

Prior research has suggested that stress can affect sleep in different ways. When people feel stressed, there will be physical and unpleasant emotional effects on them. For example, people probably experience raised blood pressure, tight muscles, frequent headaches, cold sweats, etc. From the mental perspective, people are more likely to feel overwhelmed, down and irritable. All of these effects mentioned can make people focus on and overthink their stressful events and then interfere with their regular sleep patterns. Therefore, the time to fall asleep is extended. Sleep deficiency can lead the body to maintain a constant state of inflammation, which undoubtedly raises cortisol. The level of inhibitory neurotransmitters such as serotonin decrease along with the increase of cortisol. Therefore, it is more difficult for people with low level of inhibitory neurotransmitters to fall asleep. The causal relationship between stress and sleep is like a vicious circle, which means that stress can lead to poor sleep and may thus increase the levels of stress hormones that regulate the functions of brain to make people feel even more stressed as well as harder to fall asleep. The relationship can also be indicated by the observation of EEG. In the EEG, there is a trend toward higher beta power (the dominant rhythm in patients who are alert or anxious) and decreases in delta power which occurs as people move from lighter to deeper stages of sleep [8]. Therefore, a decrease of deeper sleep, which refers to poor sleep, is accompanied by the arousal of the cortex to a higher state of alertness or tension.

\section{DATA AND METHODS}

\subsection{Data}

The research use PMData: A Sports Logging Dataset, which is a dataset that aims to combine the traditional lifelogging with sports activity logging. The data was collected by using the Fitbit Versa 2 smart watch wristband, the PMSys sports logging app and Google forms, and PMData contains logging data for 5 months from 16 persons. The mean age of the participants was $34.88(\mathrm{SD}=11.30)$ with a range from 23 to 60 and $19 \%$ female.

\subsection{Variables}

\subsubsection{Sleep duration}

Sleep duration is just a measure of how long the sleep was in hours. National Sleep Foundation has proposed sleep time duration recommendations. It suggested that the appropriate sleep duration for young adults (18-25 years) and adults (26-64 years) is between 7 and 9 hours. It should not be recommended for them to sleep for less than 6 hours and more than 11 hours [9].

\subsubsection{Sleep quality}

Sleep quality is measured with a 1- 5 scale. The score 3 is normal, and 1-2 are scores below normal and 4-5 are scores above normal.

\subsubsection{Stress}

Stress is assessed with the question: "How stressed are you?" The response categories were: highly stressed $=1, \quad$ somewhat $\quad$ stressed $=2, \quad$ normal $=3$, relieved $=4$, very relieved $=5$.

The subjective assessments of sleep duration, sleep quality and stress are reported typically once a day through a sequence of questionnaires by using the PM Reporter Pro Smartphone application.

\section{ANALYTICAL STRATEGY AND RESULTS}

The association between stress and troubled sleep is assessed by computing Pearson product-moment correlation coefficients, which indicates the direction of the association. The correlation between stress and sleep quality, stress and sleep duration were analyzed respectively. Following this, the research further used simple linear regression to reveal the effect of stress on the sleep quality and sleep duration. All analyses helped the research to find out the relationship between stress and troubled sleep.

Table 1 illustrates the descriptive statistics for all analysis variables. The mean age of the participants was $34.88(\mathrm{SD}=11.30)$ with a range from 23 to 60 and $19 \%$ female. During the five-month time, the mean stress level of the participants was 2.9, which is close to the normal level of stress, while the maximum score of stress was 5 , which represents that the participants were highly 
stressed. A similar result also occurs to the sleep quality as well as sleep duration. The mean of sleep quality was at normal value of stress, and the participant had the experience of the poorest sleep quality $(\mathrm{MIN}=0)$. On average, the participants slept for 6.7 hours a day, which is slightly shorter than the recommended sleep duration time (7-9 hours). Besides, the minimum value of sleep duration was 0 , which means that it has happened that the participant did not sleep at all.

Table1. Descriptive Statistics

\begin{tabular}{|l|c|c|c|c|c|}
\hline & SUM & MEAN & MAX & MIN & SD \\
\hline Stress & 5077 & 2.906 & 5 & 0 & 0.723 \\
\hline Sleep Quality & 5265 & 3.014 & 5 & 0 & 0.752 \\
\hline Sleep Duration & 11736 & 6.718 & 12 & 0 & 1.338 \\
\hline Age & 558 & 34.875 & 60 & 23 & 11.302 \\
\hline Gender & 19 & 1.188 & 2 & 1 & 0.390 \\
\hline
\end{tabular}

The correlations displayed in Table 2 show that the stress is positively associated with sleep quality $(\mathrm{r}=0.32)$, and the stress is also positively associated with sleep duration $(r=0.21)$. In other words, the more people feel stressed, the poorer the sleep quality is; the more people feel stressed, the shorter the sleep duration is. The simple linear regression model is presented in Figure1. The associations between dependent variables (sleep quality and sleep duration) and stress shown in Figure1 were consistent with the results in table 2 and with findings reported in previous studies.

Table2. Pearson product-moment correlation coefficients between independent and dependent variables

\begin{tabular}{|c|c|c|c|}
\hline & Stress & Sleep Quality & Sleep Duration \\
\hline Stress & & 0.3184 & 0.2069 \\
\hline
\end{tabular}

Model 1

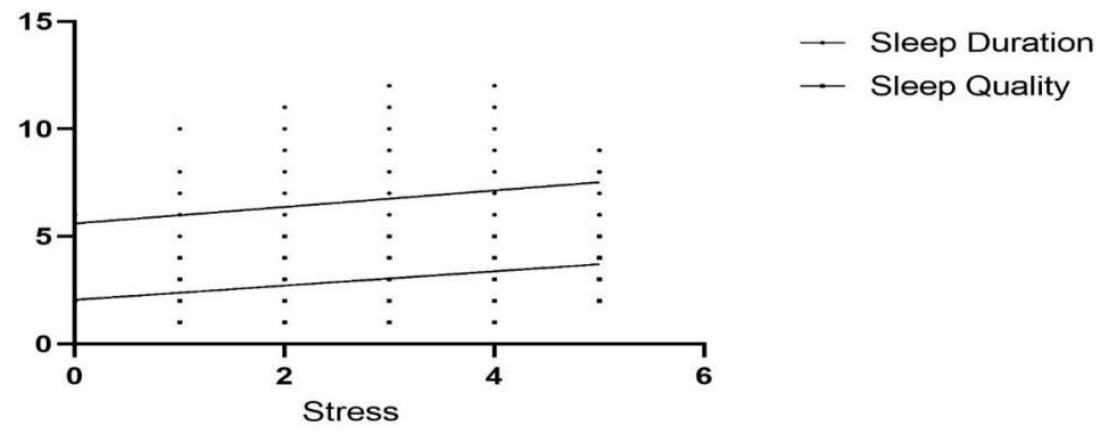

Figure1. The simple linear regression model of sleep duration, sleep quality and stress

\section{CONCLUSION}

Troubled sleep is increasingly prevalent among people all over the world. The causes of troubled sleep are plenty, and this paper is focused on the effects of stress on sleep. The stressful events and thoughts can increase the levels of stress hormones, which leads to troubled sleep. According to the analysis on the relationship between stress and troubled sleep, feeling stressed can lead to shortened sleep and poor sleep, which is consistent with the findings reported in previous studies. With the finding of the causes of troubled sleep, there will be effective methods to solve the problem.

The research used PMData, which only collected data of 16 persons, to analyze the association between stress and troubled sleep. The size of sample is relatively small, so a larger sample is required to make the correlation of 
the variables more precisely. Besides, the assessments of sleep quality, sleep duration and stress are subjective. The self-reports of data may be not as accurate as objective assessments, due to possibility of reporting errors and participants' certain concerns. In addition, the research only analyses the situation that stress is the independent variable. Some prior research has proposed that the association between sleep and stress is reciprocal. Therefore, more experiments and analyses are required to give further evidence of the situation that sleep is the independent variable.

\section{AUTHORS' CONTRIBUTIONS}

This paper is independently completed by Jiahan Wang.

\section{ACKNOWLEDGMENTS}

To begin with, I am very grateful to my teachers and professors, who provided me with valuable suggestions on my topic and organization of the paper. I still remember the discussions with my teachers, which are helpful a lot. I would like to thank all the people who gave me supports and encouragements throughout the whole process of writing the paper. Thanks to the helps and supports, the paper has been completed.

\section{REFERENCES}

[1] National Institutes of Health Sleep Disorders Research Plan 2011. https://www.nhlbi.nih.gov/files/docs/ncsdr/201101 011National Sleep Disorders Research Plan DHHS Publication,11-7820.pdf.

[2] National Sleep Foundation 2020. http://www.sleepdoundation.org/insomnia.

[3] Tiankan Zhang. Sleep and Health. 10.3969/j.issn.1008-1070.2003.08.013
[4] Sarah A. Burgard and Jennifer A. Ailshire. Putting Work to Bed: Stressful Experiences on the Job and Sleep Quality. J Health Soc Behav. 2009 December; 50(4): 476-492.

[5] T Akerstedt, A Knutsson, P Westerholm, T Theorell, L Alfredsson and G Kecklund. Sleep Disturbances, Work Stress and Work Hours: A Cross-sectional Study. Volume 53, Issue 3, September 2002, Pages 741-748.

[6] Jennifer A. Ailshire and Sarah A. Burgard. Family Relationships and Troubled Sleep among U.S. Adults: Examining the Influences of Contact Frequency and Relationship Quality. Journal of Health and Social Behavior, 53(2) 248-262.

[7] Morin, Charles M. PhD; Rodrigue, Sylvie MPs, and; Ivers, Hans MPS. Role of Stress, Arousal, and Coping Skills in Primary Insomnia. Psychosomatic Medicine, 2003, 65:259-267.

[8] Hall M, Buysse DJ, Nowell PD, Nofzinger EA, Houck P, Reynolds CF. Kupfer DJ. Symptoms of Stress and Depression as Correlates of Sleep in Primary Insomnia. Psychosomatic Medicine, 01 Mar, 2000, 62(2):227-230.

[9] National Sleep Foundation's Sleep Time Duration Recommendations: Methodology and Results Summary. Sleep Health 1, 2015: 40-43. 